\title{
Molecular docking and pharmacophore studies of heterocyclic compounds as Heat shock protein 90 (Hsp90) Inhibitors
}

\author{
Suby T Baby ${ }^{1 *}$, Shailendra Sharma1 ${ }^{1}$, Sreenivas Enaganti², Roby P. Cherian ${ }^{3}$ \\ 1Faculty of Pharmaceutical Sciences, Jodhpur National University, Jodhpur, Rajasthan 342003, India; 2Bioinformatics division, Averin \\ biotech, Nallakunta, Hyderabad, Telangana 500044, India; 3Jazan University, P.O.Box 114, Jazan 45142, Kingdom of Saudi Arabia. Suby \\ T Baby - Email: subysusanbaby@yahoo.com; *Corresponding author
}

Received April 9, 2016; Revised May 15, 2016; Accepted May 16, 2016; Published June 15, 2016

\begin{abstract}
:
Heat Shock Protein 90 was a key molecular chaperone involved in the proteome stability maintenance and its interference in many signaling networks associated with cancer progression, makes it of an important target for cancer therapeutics. The present study aimed to identify potential lead molecule among the selected heterocyclic compounds against Human Hsp90 (PDB: 1YET) through docking using GOLD 3.1 and pharmacophore studies using Discovery studio 2.1. On the basis of the GOLD Fitness scores, the compounds Q1G and T21 showed better binding affinity. Further the analyzed structure pharmacophore results are in consistence with the docking results indicating that both these compounds show antagonistic activity towards HSP90 respectively.
\end{abstract}

Keywords: molecular chaperone, Heat Shock Protein 90, docking, pharmacophore, cancer, proteome

\section{Background:}

Hsp90 was a well conserved molecular chaperone required to maintain internal stability of the proteins and keep up viability of the cells under stressed conditions [1]. Additionally under normal conditions, it plays a leading role in organizing the stability and activation state of a variety of 'client' proteins, many of which are important in mediating several fundamental cellular pathways such as signal transduction, cell cycle progression, transcription regulation and the modulation of immunogenic proteins $[2,3,4]$. Accompanying these client proteins depend upon a dynamic cycle directed by ATP binding to Hsp90 and consequential hydrolysis [5]. Also, the collaboration of Hsp90 with different considerable other co-chaperones attains its complete functional activity. This collaboration regulates various activities through ATPase activation and inhibition besides recruitment of client proteins to the cycle precisely [6]. The Hsp90 client proteins differ both in their structure and function ranging from telomerase to kinases and transcription factors $[1,7,8]$. Amongst the client proteins of Hsp90 are many oncogenes such as Her2/ErbB2, Akt, Raf-1, Hif-1 , hormone receptors, survivin, mutant p53 and hTERT which mediate survival, proliferation, invasion, metastasis and angiogenesis of tumors $[9,10,11]$. They behave as key regulators of cellular growth, differentiation, stress-response and apoptotic pathways [12]. Besides these, 
abnormal expression of Hsp90 has been implicated in a variety of disease states importantly in cancer where the chaperoning of mutated and over expressed oncoproteins was critical. In cancer, raised Hsp90 levels induce the stability and function of oncogenic proteins found through all six hallmarks of cancer such as angiogenesis, immortalization, metastasis, impaired apoptosis, insensitivity to antigrowth signals, autocrine growth $[\mathbf{1 3}, \mathbf{1 4}, \mathbf{1 5}$. Therefore, pharmacological inhibition of Hsp90 as a "druggable" target for rational cancer therapy has been of much interest because of its simultaneous interruption of multiple signaling pathways associated with cancer progression [16]. Thus targeting Hsp90 with specific chemical inhibitors leads directly to the down regulation of client proteins and attains antiproliferative activity through the arrest of the tumour growth, morphological and functional differentiation, and activation of apoptosis [17]. Given the potential of Hsp90 as an anticancer target, a better understanding of Hsp90 functions may facilitate the design of better therapeutic strategies.<smiles>C=C1NC(c2cc(OC)c(OC)c(OC)c2)=CN1Cc1ccc(Cl)cc1</smiles>

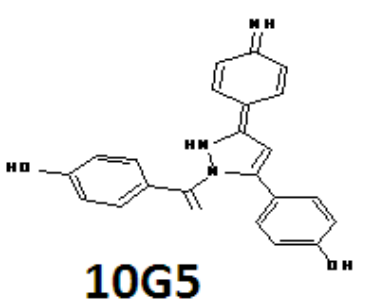<smiles>O=C1C=C(O)C=CC1=C(O)CSc1nc2ccc(Cl)cc2c(=O)n1-c1ccccc1</smiles>

Q10<smiles></smiles>

QB44<smiles>COc1cc(-c2cn(Cc3ccc(O)cc3)c(=S)[nH]2)cc(O[GaH])c1OCc1ccccc1</smiles><smiles>COc1ccc(C2=CC(=C3C=CC(=O)C=C3)N(C(=O)c3ccc(O)cc3)[NH2+]2)c(OC)c1</smiles>

10G14

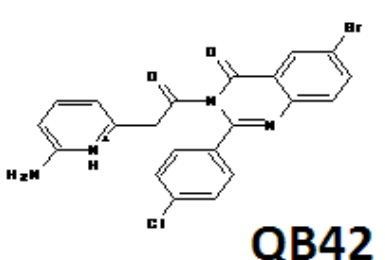

QB42<smiles></smiles>

P2b

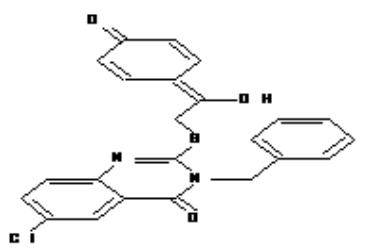

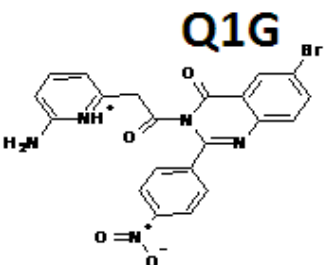

QB43

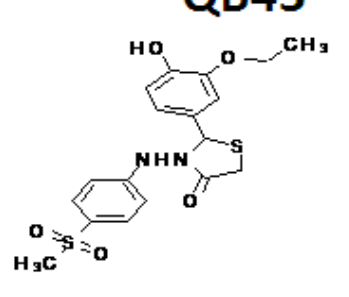

T14

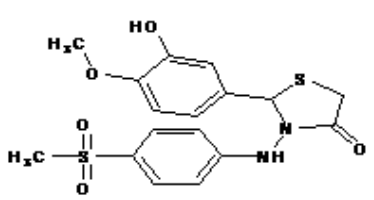

T20

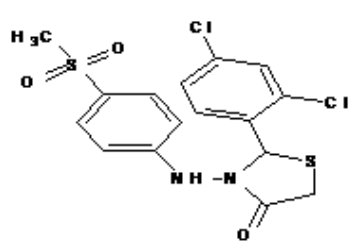

T7

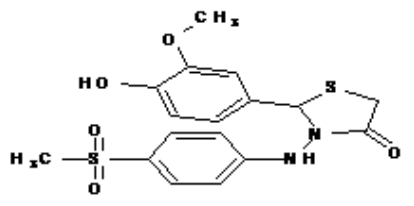

T21

Figure 1: 2D structures of the compounds used in this study. 


\section{Open access}

In the race of new anticancer drug development, heterocyclic derivatives, due to their widespread therapeutic uses, have attracted a great deal of attention amongst the scientific community. Heterocycles can be easily manipulated to achieve the required modification in function and their moieties are part of compounds showing numerous biological activities like antibacterial, anti-viral, anti-fungal, anti-inflammatory, and antitumor drugs [18-20]. Based on the principle of structural diversity and wide coverage of activity range reported in various literature resources [21-27], heterocyclic compounds were carefully selected which showed anticancer properties. Herein, our study we propose to investigate the anticancer activity of those selected Heterocyclic derivatives against the target protein Hsp90, confirm their antagonist activity by molecular docking and pharmacophore studies.

\section{Methodology: \\ Protein preparation}

The crystal structure of Human Hsp90-Geldanamycin binding domain (PDB: 1YET) was retrieved from the Protein Data Bank and imported into Accelrys Discovery Studio 2.5. Water molecules and complexes bound to receptor molecule were removed. Further using the clean Protein protocol within Discovery studio, protein preparation was carried out with the addition of hydrogen atoms to the protein for correct ionization and tautomeric states of amino acid residues. Missing loops, missing atoms were modeled and alternate conformations were removed for refining crystallographic disorder. Following the above steps of preparation, the protein was subjected to energy minimization by applying the CHARMm forcefield using steepest descent algorithm with a maximum number of 1000 steps at RMS gradient of 0.01 . This was continued until the protein satisfied with a convergence gradient of $0.001 \mathrm{kcal} \mathrm{mol}^{-1}$.

\section{Generation of ligand dataset}

The selected heterocyclic derivatives from various literature resources [21-27] were drawn using ACD/ChemSketch (12.0). Figure 1 shows the 2D structure of the sketched compounds. Further imported into Accelrys Discovery Studio 2.1 and ligand preparation with constraint parameters such as consistency of ionization states, tautomer and isomer generation, removal of duplicate structures, conversion of 2D to 3D structures was done. By applying the forcefield CHARMm, minimization was carried out with the smart minimizer algorithm till it satisfied with the convergence gradient of $0.001 \mathrm{kcal} \mathrm{mol}^{-1}$, to attain the lowest energy conformers which were taken for further evaluation.

\section{Molecular Docking Studies \\ Docking strategy}

A primary objective in molecular docking was the ability to estimate the scoring function and evaluate protein-ligand interactions in order to predict the binding affinity and activity of the ligand molecule. The docking program GOLD have been employed to generate the bioactive binding poses of heterocyclic derivatives in the active site of protein Hsp90. GOLD 3.1 (Genetic
Optimization for Ligand Docking; Cambridge Crystallographic Data Centre) uses a genetic algorithm to explore the conformational space of the ligands in addition to some flexibility of active site residues. Protein coordinates from the bound ligand of Hsp90 were used to define the active site. Docking calculations were performed using the default GOLD fitness function and default GOLD parameters were used to produce the set of optimal conformations of both the ligand and the protein. Each simulation was performed 10 times; yielding 10 docked conformations unless three of the 10 poses were within $1.5 \AA$ RMSD of each other. The lowest energy conformations were regarded as the binding conformations between ligands and the protein. Greater the GOLD fitness score better the binding affinity. Hit molecules which showed the expected interactions with the critical amino acids present in the active site of the protein, may show potent antagonist properties towards Hsp90.

\section{Pharmacophore modeling}

\section{Generation of structure-based pharmacophore model}

To further identify the critical structural features of the protein that was important for the ligand binding, structure-based pharmacophore modeling was employed based on the protein ligand complexes. This molecular modeling was carried out to construct a hypothetical pharmacophore model aiming to study fitting of the designed compounds to the generated pharmacophores of our target protein Human Hsp90. All pharmacophore modeling studies was performed using Catalyst in DS.

To build the pharmacophore model for screening, corresponding possible interaction points from active site of Human Hsp90 protein (PDB: 1YET) was generated using the 'Interaction Generation' protocol implemented in Discovery Studio. It extracted all the available hydrophilic and lipophilic interaction points that can be complemented by the inhibitor. The parameters for both 'Density of Lipophilic Sites' and 'Density of Polar Sites' were defined as 10 . The active site was then analysed for hydrogen bond donors, acceptors, and hydrophobic features by generating pharmacophore query from the Ludi interaction maps. During pharmacophore generation a minimum of 1 and a maximum of 2 pharmacophore feature such as hydrogen bond donor (HBD), hydrogen bond acceptor (HBA), and hydrophobic (HY) were included. Using Edit and Cluster pharmacophore tools, these features were then clustered and the most representative features with catalytic importance were selected and included in the pharmacophore hypothesis. After these operations, a structure-based pharmacophore model comprising the most important pharmacophoric features was built. The final pharmacophore model was subjected to validation based on the active site orientation of inhibitors.

\section{Validation of the Pharmacophore}

To validate the generated structure-based pharmacophore model, the heterocyclic derivatives are mapped onto the pharmacophore model. This was done by Ligand pharmacophore mapping 
protocol of DS with the Best Flexible Search option for conformational analysis of each compound with low energies. Maximum Omitted Features option was chosen as 2 because mapping all the features present in the structure-based hypothesis will reduce the hit rate. The predictive ability of the model was analyzed based on the best fit values that indicate how well the compounds were mapped onto the pharmacophoric features. Compounds were ranked based on fit values. A higher fit value represents a better fit.

\section{Results \& Discussion:}

\section{Molecular docking studies}

To find the binding affinities and key interactions between the HSP90 and heterocyclic derivatives docking studies was carried out using GOLD. Two important parameters have been considered for selecting potential compounds among the given input: (i) prediction of binding energy of the best docked pose using scores calculated by GOLD scoring function and (ii) Hydrogen bond details of the top-ranked pose. The docking run generated 10 different poses for each of the compound and the corresponding GOLD fitness scores were generated. Higher the fitness score of the ligand pose was better because it was calculated based on the negative of the sum of the component energy terms. The fitness function was first optimized for the prediction of ligand binding position. The well orientated ligand pose have lowest energy with average Gold fitness scores were enumerated. So, the above pre-validated analysis was used to sort out the retrieved hit molecules and then those are further validated by using the visualization method to find the suitable binding mode of the inhibitors based on the critical interactions with the active site residues. The Summary of docking information of the top ranked poses was tabulated in Table 1.

Table 1: Docking scores of the compounds with Human Hsp90 (PDB: 1YET)

\begin{tabular}{|c|c|c|c|c|c|c|}
\hline Compound name & $\begin{array}{l}\text { No. of } \\
\text { rotatable } \\
\text { bonds }\end{array}$ & $\begin{array}{l}\text { Fitness } \\
\text { score }\end{array}$ & S(hb_ext) & S(vdw_ext) & S(hb_int) & S(int) \\
\hline $\begin{array}{l}\text { 1-(4-chlorobenzyl)-4-(3,4,5-trimethoxy phenyl)-1,3-dihydro-2H- } \\
\text { imidazole-2-thione (I2a) }\end{array}$ & 6 & 71.45 & 0.00 & 57.62 & 0.00 & -7.77 \\
\hline $\begin{array}{l}\text { 1-(4-hydroxybenzyl)-4-(3,4,5-trimethoxy phenyl)-1,3-dihydro-2H- } \\
\text { imidazole-2-thione (I2g) }\end{array}$ & 6 & 72.40 & 8.12 & 54.14 & 0.00 & - \\
\hline $\begin{array}{l}\text { 4- }(5-\{[(E)-(\{(E) \text {-hydroxy }[4- \\
\text { (trifluoromethyl)phenyl]methylidene }\} \text { amino) (sulfanyl) } \\
\text { methylidene]amino }\}-1 H \text {-pyrazol-1-yl)phenolate }(\mathrm{PZb})\end{array}$ & 5 & 69.57 & 1.13 & 54.53 & 0.00 & -6.53 \\
\hline $\begin{array}{l}\text { 4-\{[3-(4-aminophenyl)-5-(4-hydroxy phenyl)-1H-pyrazol- } \\
\text { 1yl](hydroxy) methylidene\} cyclohexa-2,5-dien-1-one (10G5) }\end{array}$ & 2 & 67.29 & 9.56 & 51.50 & 0.00 & 13.09 \\
\hline $\begin{array}{l}4-\{[3-(2,4-d i m e t h o x y p h e n y l)-5-(4-h y d r o x y p h e n y l)-1 H \text {-pyrazol-1-yl] } \\
\text { carbonyl\}phenolate (10G14) }\end{array}$ & 4 & 68.98 & 11.70 & 49.44 & 0.00 & $\overline{1}-71$ \\
\hline $\begin{array}{l}\text { 3-benzyl-6-chloro-2-\{[2-(4-hydroxyphenyl)-2- } \\
\text { oxoethyl]sulfanyl\}quinazolin-4(3H)-one } \\
\text { (Q1g) }\end{array}$ & 5 & 81.19 & 7.17 & 60.73 & 0.00 & -9.49 \\
\hline $\begin{array}{l}\text { 6-chloro-2-\{[(2E)-2-hydroxy-2-(2-hydroxy-4-oxocyclohexa-2,5-dien-1- } \\
\text { ylidene)ethyl] sulfanyl\}- 3-phenylquinazolin-4(3H)-one } \\
\text { (Q1o) }\end{array}$ & 4 & 71.58 & 6.25 & 52.84 & 0.00 & -7.33 \\
\hline $\begin{array}{l}\text { 3-[(6-aminopyridin-2-yl)acetyl]-6-bromo-2-(4- } \\
\text { chlorophenyl)quinazolin-4(3H)-one (QB42) }\end{array}$ & 3 & 70.32 & 4.73 & 54.90 & 0.00 & -9.91 \\
\hline $\begin{array}{l}\text { 3-[(6-aminopyridin-2-yl)acetyl]-6-bromo-2-(4-nitrophenyl)quinazolin- } \\
\text { 4(3H)-one } \\
\text { (QB43) }\end{array}$ & 4 & 68.79 & 4.65 & 55.69 & 0.00 & - \\
\hline $\begin{array}{l}\text { 3-[(6-aminopyridin-2-yl)acetyl]-6-bromo-2-(4- } \\
\text { methoxyphenyl)quinazolin-4(3H)-one (QB44) }\end{array}$ & 4 & 68.44 & 4.75 & 55.37 & 0.00 & - \\
\hline $\begin{array}{l}(2 S)-2-(2,4-d i c h l o r o p h e n y l)-3-\{[4-(\text { methylsulfonyl)phenyl }] \text { amino }\}-1,3- \\
\text { thiazolidin-4-one (T7) }\end{array}$ & 4 & 67.17 & 0.00 & 50.74 & 0.00 & -2.60 \\
\hline $\begin{array}{l}\text { (2S)-2-(3-ethoxy-4-hydroxyphenyl)-3-\{[4- } \\
\text { (methylsulfonyl)phenyl]amino\}-1,3-thiazolidin-4-one (T14) }\end{array}$ & 4 & 69.02 & 7.45 & 49.93 & 0.00 & -7.09 \\
\hline $\begin{array}{l}\text { (2S)-2-(3-hydroxy-4-methoxyphenyl)-3-\{[4- } \\
\text { (methylsulfonyl)phenyl]amino\}-1,3-thiazolidin-4-one (T20) }\end{array}$ & 4 & 70.09 & 8.37 & 49.03 & 0.00 & -5.70 \\
\hline $\begin{array}{l}\text { (2S)-2-(4-hydroxy-3-methoxyphenyl)-3-\{[4- } \\
\text { (methylsulfonyl)phenyl]amino\}-1,3-thiazolidin-4-one (T21) }\end{array}$ & 4 & 70.14 & 7.50 & 50.51 & 0.00 & -6.81 \\
\hline $\begin{array}{l}\text { 2-phenyl-3-[5-(2-phenylethenyl)-1,3,4-thiadiazol-2-yl]-1,3-thiazolidin- } \\
\text { 4-one } \\
\text { (T2h) }\end{array}$ & 4 & 68.25 & 0.16 & 51.57 & 0.00 & -2.81 \\
\hline
\end{tabular}




\section{Open access}

From the overall docking scores we identified that all the compounds were having the approximate Gold fitness scores. The stability of the best docked pose of these compounds was evaluated by determining the hydrogen bonding interactions of the protein with compounds which revealed the critical amino acids involved in hydrogen bond formation. Based on these factors, among all the compounds Q1g was having the highest fitness score of 81.19 indicating high binding affinity and better hydrogen bond interactions with the Hsp90 (1YET) active site residues. From Figure 2a it was revealed that the compound Q1g formed three hydrogen bonds with protein Hsp90 (1YET). Single hydrogen bonds were formed between the compound Q1g and each of the amino acids ASP93, SER52 and TYR139 with hydrogen bond distances of $2.411 \mathrm{~A}^{\circ}, 1.719 \mathrm{~A}^{\circ}$ and $2.787 \mathrm{~A}^{\circ}$ respectively. It also formed some non-bonded interactions with the amino acid PHE 162 with a distance of $2.886 \mathrm{~A}^{\circ}$. The interacting amino acids were ASP93, SER52, TYR139 and PHE 162.

Even though the compounds I2g (72.40), Q1o (71.58) , I2a (71.45), QB42 (70.32) were having high fitness scores than the compound T21 (70.14), but these compounds were not forming good hydrogen bonding interactions with Hsp90 than T21 which have formed better hydrogen bond interactions with the catalytically important residues of Hsp90. So here we considered the compound T21 as the molecule next to the compound Q1g having better antagonistic properties than the others. From Figure $\mathbf{2 b}$, it was revealed that compound T21 formed three hydrogen bonds with protein Hsp90 (1YET). A single hydrogen bond was formed between the compound T21 and amino acid TYR139 with a distance of $2.861 \mathrm{~A}^{\circ}$. Two hydrogen bonds are formed with the amino acid ASP93 and the compound T21 with a distance of $3.172 \mathrm{~A}^{\circ}$ and $3.409 \mathrm{~A}^{\circ}$ respectively and a non bonded interaction was formed with the amino acid TRP161 with a distance of $2.565 \mathrm{~A}^{\mathrm{o}}$. The interacting amino acids are TYR139, ASP93 and TRP 161. Further we validated these docking results using structure based pharmacophore mapping.

\section{Pharmacophore modeling}

For a better understanding of the key features that are responsible for biological function of our compounds, structure based pharmacophore modeling was performed. The ligand interactions with the amino acid residues present in the active site was a suitable input to design the structure based pharmacophore model of our target protein Human Hsp90 (1YET).

The Interaction Generation protocol constructed ten pharmacophore models comprising hydrogen bond acceptor (HBD), hydrogen bond donor (HBA) and hydrophobic features for our protein evaluating them based on their selectivity score, the higher the better was taken for further analysis. Using the Edit and Cluster Pharmacophore tool available in Discovery studio, the identified features are grouped and refined. The final edited pharmacophore model of Hsp90 (1YET) has two HBD, two
HBA and two hydrophobic features. The generated structure based pharmacophore model was depicted in the Figure 3.

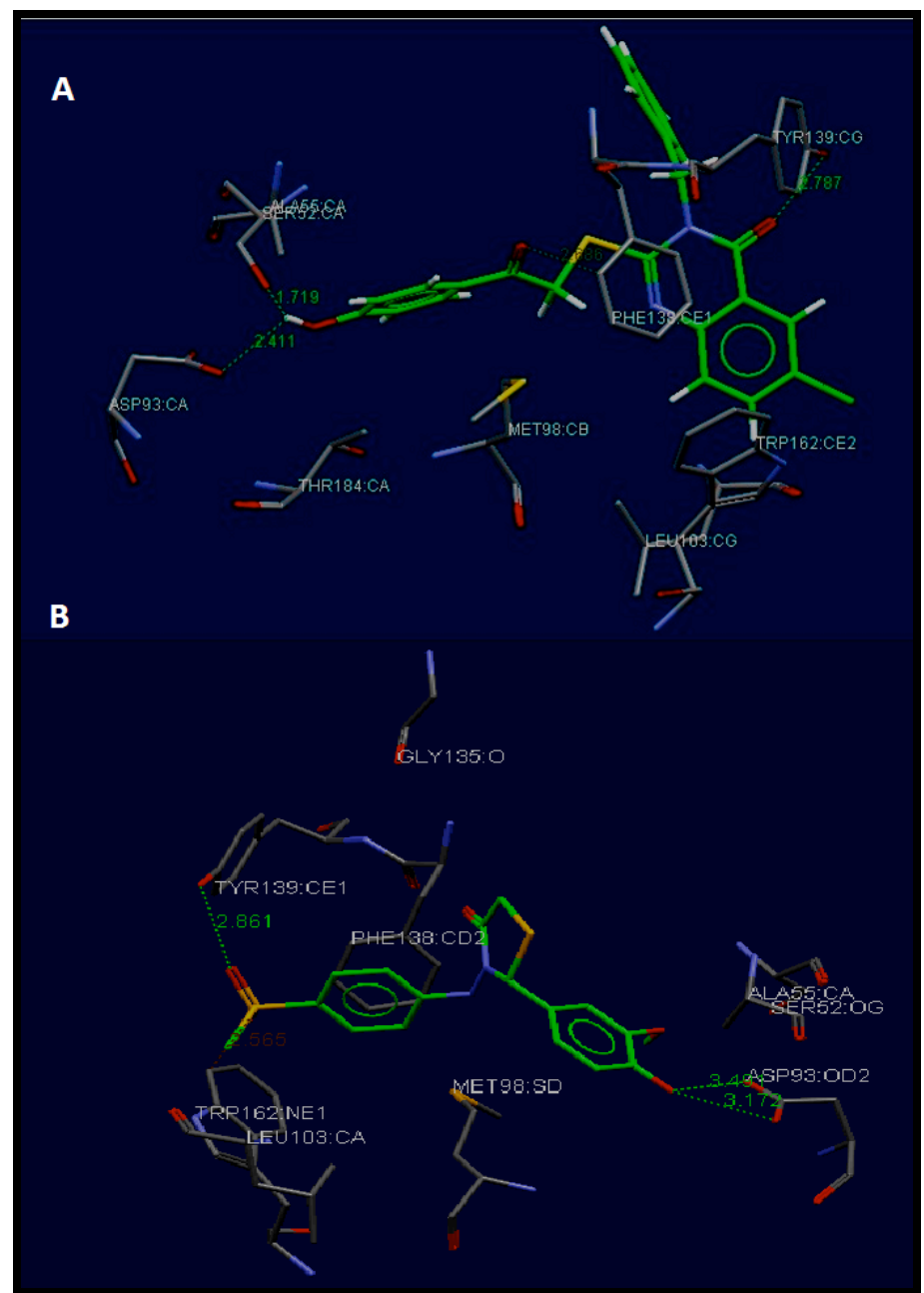

Figure 2: Hydrogen bond interactions of (a) compound Q1g and (b) compound T21 with Human Hsp90 (PDB: 1YET). The green dotted lines represents the hydrogen bonds formations and white letters showing the amino acids involved in the bonding and compounds are shown in stick model.

For the assessment of the quality of the generated pharmacophore, the final pharmacophore model undergoes validation process in accordance with the active site coordination of our compounds. At this step, it was important to find candidate compounds that being able to fit in the generated structure-based pharmacophore model, which was a reflection of the active site geometry. Using Ligand Pharmacophore Mapping protocol, with the Best conformation generation and Flexible fitting methods, all the taken heterocyclic derivative compounds are mapped on to the pharmacophore model. Geometric fit values are calculated for every compound hit based on how well the chemical substructures of a compound map on to the 


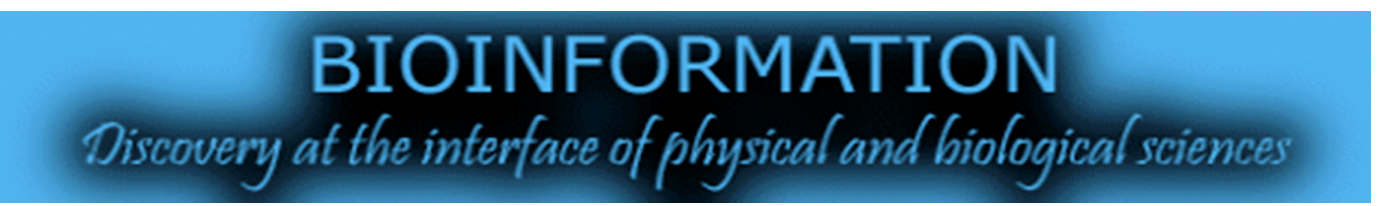

\section{Open access}

pharmacophoric feature location constraints. The best-fit value of the molecule to the respective pharmacophore was calculated. Molecules are ranked based on their fit values computed and the compounds with high fit values are chosen for further studies. The fitness scores of the compounds with the pharmacophore model of Human Hsp90 (1YET) was shown in Table 2, ranging from 3.102 to 1.994 . Here based on the fit scores, the compounds Q1g and T21 fitted well on the generated pharmacophore with fit values of 3.102 and 2.989 respectively. Mapping of the compound Q1g and T21 on to the pharmacophore model of Human Hsp90 (1YET) was shown in Figure 4.

\section{Conclusion:}

In the present study, based on the selected target protein Human Hsp90 (PDB: 1YET), the heterocyclic derivatives was analyzed for their anti cancer activity through docking and pharmacophore studies. The analyzed results of docking showed that the binding affinity of compounds Q1G and T21 with our target protein was reliable, showing significant interactions, suggesting their inhibitory activity. The pharmacophore results also indicate that the compounds were well matched with the obtained pharmacophore model features with good fit values. This study revealed that the compounds Q1G and T21 may be a potential inhibitor of Human Hsp90 as targeted for cancer, to act as a drug candidate. This work can be further evaluated experimentally through cell line studies in order to confirm it as promising in future.

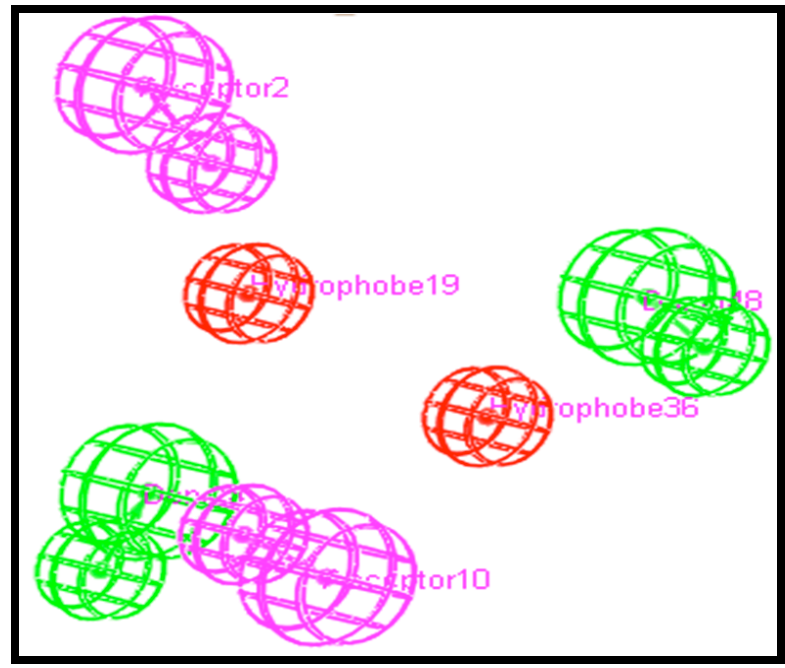

Figure 3: Generated structure based pharmacophore model of Human Hsp90 (PDB: 1YET). Green color indicates hydrogen bond acceptor (HBA); cyan indicates hydrophobic $(\mathrm{H})$ and magenta indicates hydrogen bond donor (HBD).

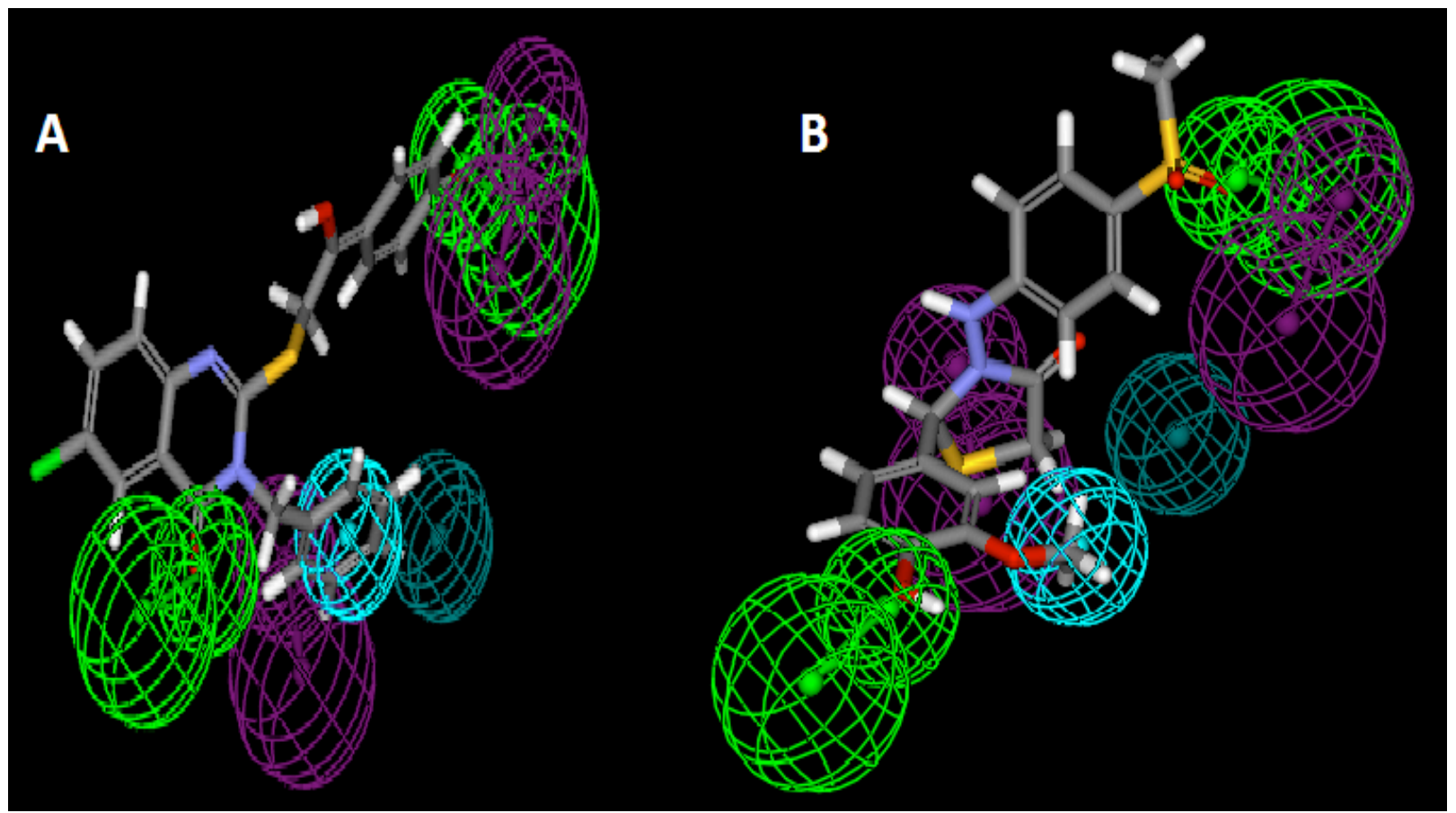

Figure 4: Ligand pharmacophore mapping of the high active compounds (a) Q1g and (b) T21 on to the structure based pharmacophore model. 


\section{BIOINFORMATION}

\section{Discovery at the interface of physical and biological sciences}

\section{Open access}

Table 2: The predicted fit values of the compounds from the structure based pharmacophore model of Human Hsp90

\begin{tabular}{llllllll} 
Name & Acceptor16 & Acceptor28 & Donor5 & Donor62 & Hydrophobe21 & $\begin{array}{l}\text { Hydrophobe } \\
\text { 43 }\end{array}$ & $\begin{array}{l}\text { Fit } \\
\text { Value }\end{array}$ \\
\hline QB42.cdx & 0 & 1 & 0 & 0 & 1 & 1 & 2.922 \\
Q1g.mol & 0 & 1 & 0 & 0 & 1 & 1 & 3.102 \\
PZb.mol & 0 & 1 & 0 & 0 & 1 & 1 & 2.902 \\
QB44.cdx & 0 & 1 & 0 & 0 & 1 & 1 & 2.894 \\
Q10.mol & 0 & 1 & 1 & 0 & 1 & 0 & 2.891 \\
QB43.cdx & 0 & 1 & 1 & 0 & 1 & 0 & 2.867 \\
10G14.cdx & 0 & 1 & 0 & 0 & 1 & 1 & 2.805 \\
I 2g.mol & 0 & 1 & 0 & 0 & 1 & 1 & 2.779 \\
T14.cdx & 1 & 1 & 0 & 0 & 1 & 0 & 2.715 \\
I 2a.mol & 0 & 1 & 0 & 0 & 1 & 1 & 2.713 \\
T21.cdx & 1 & 1 & 0 & 0 & 1 & 0 & 2.989 \\
T20.cdx & 1 & 1 & 1 & 0 & 0 & 0 & 2.539 \\
T7.cdx & 1 & 1 & 0 & 0 & 1 & 0 & 2.408 \\
T2h.mol & 0 & 1 & 0 & 0 & 1 & 0 & 1.998 \\
10G5.cdx & 1 & 1 & 0 & 0 & 0 & 0 & 1.994 \\
\hline
\end{tabular}

\section{References:}

[1] Taipale M et al. Nat Rev Mol Cell Biol. 2010 11:515 [PMID: 20531426]

[2] Richter K \& Buchner J, J Cell Physiol. 2001 188:281 [PMID: 11473354]

[3] Was aacs JS et al. Cancer Cell. 2003 3:213 [PMID: 12676580]

[4] Pratt WB \& Toft DO, Exp Biol Med (Maywood) 2003 228:111 [PMID: 12563018]

[5] Neckers L, Curr Med Chem. 2003 10:733 [PMID: 12678776]

[6] Sreedhar AS et al. Biochim Biophys Acta. 2004 1697:233 [PMID: 15023364]

[7] Zhao R et al. Cell. 2005 120:715 [PMID: 15766533]

[8] Taipale M et al. Cell. 2012 150:987 [PMID: 22939624]

[9] Sharp S \& Workman P, Adv Cancer Res. 2006 95:323 [PMID: 16860662]

[10] Neckers L \& Ivy SP, Curr Opin Oncol. 2003 15:419 [PMID: 14624223]

[11] Fortugno P et al. Proc Natl Acad Sci U S A. 2003 100:13791 [PMID: 14614132]

[12] Lanneau D et al. J Cell Mol Med. 2008 12:743 [PMID: 18266962]

[13] Hanahan D \& Weinberg RA, Cell. 2011 144:646 [PMID: 21376230].

[14] Whitesell L \& Lindquist SL, Nat Rev Cancer. 2005 5:761 [PMID: 16175177]
[15] Workman P et al. Ann N Y Acad Sci. 2007 1113:202 [PMID: 17513464]

[16] Blagg BS \& Kerr TD, Med Res Rev. 2006 26:310 [PMID: 16385472]

[17] Marcu MG et al. J Natl Cancer Inst. 2000 92:242 [PMID: 10655441]

[18] Arora P et al. Int J Pharm Res Sci. 2012 3:2947

[19] Nagalakshmi G, Indian J Pharm Sci. 2008 70:49 [PMID: 20390080]

[20] Sperry JB \& Wright DL, Curr Opin Drug Discov Devel. 2005 8:723 [PMID: 16312148]

[21] Avila C et al. Bioorg Med Chem Lett. 2006 16:3005 [PMID: 16530412]

[22] Sakkiah S et al. Eur J Med Chem. 2011 46:2937 [PMID: 21531051]

[23] Sakkiah S et al. Eur J Med Chem. 2010 45:2132 [PMID: 20206418]

[24] Brough PA et al. Bioorg Med Chem Lett. 2005 15:5197 [PMID: 16213716]

[25] Cheung KM et al. Bioorg Med Chem Lett. 2005 15:3338 [PMID: 15955698]

[26] Joseph A et al. Acta Pharm. 2013 63:397 [PMID: 24152899]

[27] Park H et al. Bioorg Med Chem Lett. 2007 17:6345 [PMID: 17869098]

Edited by P Kangueane

Citation: Baby et al. Bioinformation 12(3): 149-155 (2016)

License statement: This is an Open Access article which permits unrestricted use, distribution, and reproduction in any medium provided the original work is properly credited. This is distributed under the terms of the Creative Commons Attribution License

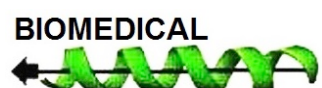

INFORMATICS
ISSN 0973-2063 (online) 0973-8894 (print)
155

BIOMEDICAL

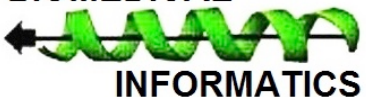

